\title{
Sobrecarga de familiares cuidadores em relação ao diagnóstico de pacientes psiquiátricos
}

\author{
Family caregivers' burden as a function of psychiatric patients diagnostics
}

Marcela Nolasco ${ }^{1}$, Marina Bandeira², Marcos Santos de Oliveira ${ }^{3}$, Carlos Eduardo Leal Vidal ${ }^{4}$

\section{RESUMO}

Objetivo: Comparar os graus das sobrecargas objetiva e subjetiva sentidas por familiares cuidadores de pacientes com esquizofrenia e por familiares cuidadores de pacientes com depressão maior, bem como os fatores associados e as dimensões mais afetadas em cada grupo. Métodos: Participaram desta pesquisa 50 cuidadores de pacientes com esquizofrenia e 50 cuidadores de pacientes com depressão maior. Esses familiares participaram de uma entrevista estruturada, na qual foram aplicados dois instrumentos: a escala de sobrecarga FBIS-BR e um questionário. Resultados: Os resultados indicaram que os dois grupos apresentavam diferenças significativas quanto ao grau de sobrecarga, na análise detalhada dos itens da escala. Os familiares cuidadores de pacientes com esquizofrenia apresentaram sobrecarga objetiva significativamente mais elevada ao assistir o paciente na tomada de medicamentos e na administração do dinheiro e apresentaram maior sentimento de peso financeiro resultante do papel de cuidador. Os cuidadores de pacientes com depressão maior apresentaram maior frequência de supervisão de comportamentos autoagressivos, mais preocupação com a vida social dos pacientes e maior sentimento de incômodo nas tarefas de assistência na vida cotidiana. Não foram encontrados dados significativos referentes aos escores globais da escala de sobrecarga. Conclusão: As diferenças encontradas nesta pesquisa apontam para a necessidade de os serviços de saúde mental planejarem intervenções específicas para cada grupo de cuidadores.

\section{ABSTRACT}

Objective: This study aimed to compare the degree of objective and subjective burden felt by family caregivers of patients with schizophrenia and by family caregivers of patients with major depression, as well as the associated factors and life dimensions most affected in each group. Methods: Participated in this study 50 family caregivers of patients with schizophrenia and 50 family caregivers of patients with major depression. These subjects participated in a structured interview for the application of the FBIS-BR burden scale and a sociodemographic and clinical questionnaire. Results: The results indicated significant differences in the degree of burden of the two family caregivers groups, in the analysis of specific scale items. The family caregivers of patients with schizophrenia showed significantly higher ob-

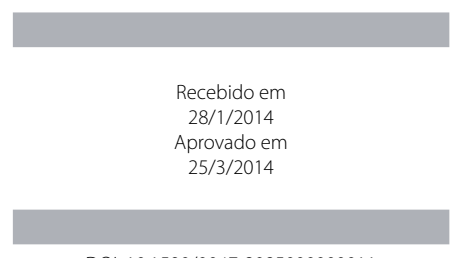

DOI: $10.1590 / 0047-2085000000011$
1 Universidade Federal de São João del-Rei (UFSJ), Programa de Mestrado em Psicologia, Linha de Pesquisa em Saúde Mental. UFSJ, Laboratório de Pesquisa em Saúde Mental (LAPSAM).

2 UFSJ. Conselho Nacional de Desenvolvimento Científico e Tecnológico (CNPq). Université de Montréal, Canadá. LAPSAM.

3 UFSJ. Universidade Federal de Lavras (UFLA).

4 Secretaria de Estado da Saúde. Universidade Federal de Minas Gerais (UFMG), Faculdade de Medicina.

Endereço para correspondência: Marcela Nolasco

Rua Américo Pereira Campos, 57

36300-190 - São João del-Rei, MG, Brasil

Telefones: (32) 3371-8646/(32) 8885-8646

E-mail: marcela.nolasco@hotmail.com 


\section{Keywords}

Family caregivers, family

caregivers' burden,

psychiatric patients,

patients' diagnostics,

schizophrenia and major

depression. jective burden in the subscale "Assistance in Everyday Life", particularly in the tasks related to medication administration and financial management, and they also had more feeling of financial burden. Caregivers of patients with major depression had a higher frequency of self-injurious behaviors supervision, more concern with the social life of patients and a greater feeling of discomfort in the service tasks in everyday life. No difference was observed between groups regarding the scale global scores. Conclusion: The differences found in this study pointed out the need for mental health services to plan specific interventions for each group of family caregivers.

\section{INTRODUÇÃO}

O tratamento dos transtornos psiquiátricos passou por modificações importantes desde a desinstitucionalização psiquiátrica desenvolvida em diversos países nas últimas décadas. Esse processo se caracterizou pela redução progressiva dos leitos psiquiátricos nos hospitais e pela ênfase nos serviços comunitários, colocando em destaque a reinserção social dos pacientes na comunidade, a promoção da sua qualidade de vida e o envolvimento das famílias ${ }^{1-4}$. As famílias tornaram-se as principais provedoras de cuidados e apoio aos pacientes. Como resultado, ocorreu a sobrecarga dos familiares, definida como o sentimento de peso a carregar por causa do papel de cuidadores dos pacientes e das dificuldades que encontraram no desempenho desse papel em seu dia a dia ${ }^{5}$. O uso do termo sobrecarga familiar refere-se somente aos efeitos do transtorno psiquiátrico na família, e não à pessoa do paciente. Segundo Hatfield, os familiares passaram a prestar cuidados aos pacientes em uma base de 24 horas, o que era feito anteriormente, nas instituições, por três equipes de funcionários em turnos de 8 horas $^{6}$. Esse autor destacou que a desinstitucionalização transferiu essas responsabilidades aos membros da família após terem decorridos 100 anos de institucionalização psiquiátrica, nos quais as instituições haviam assumido integralmente o atendimento aos pacientes. Entretanto, o compromisso das famílias em assumir o papel de cuidadores em tempo integral era limitado e os familiares não foram preparados para esse papel $\left.\right|^{5}$.

O conceito de sobrecarga foi definido em dois aspectos: objetiva e subjetiva. A sobrecarga objetiva envolve as tarefas cotidianas de assistência aos pacientes, as perdas financeiras e as perturbações na rotina, na vida social e na vida profissional dos familiares ${ }^{7}$. A sobrecarga subjetiva é definida pelo sentimento de peso a carregar na função de cuidador, assim como pelas emoções e preocupações que os cuidadores apresentam em relação ao paciente ${ }^{8,9}$. Maurin e Boyd ${ }^{10}$ propuseram um modelo teórico que sintetiza as relações entre as variáveis que afetam a sobrecarga subjetiva dos cuidadores. Esse modelo considera que a sobrecarga subjetiva seria resultante da interação de três níveis de variáveis. As variáveis antecedentes são aquelas que definem a situação do transtorno psiquiátrico, envolvendo o diagnóstico, os sintomas e o grau de autonomia do paciente, além do tipo de residência e as características do programa de tratamento no qual o paciente está inserido. Essas variáveis determinam o grau de sobrecarga objetiva, pois afetam a frequência das tarefas cotidianas necessárias para cuidar do paciente, a frequência das supervisões aos comportamentos problemáticos dos pacientes e as interferências na vida social e profissional do cuidador, tais como sintomas e nível de autonomia do paciente. As variáveis antecedentes, juntamente com a sobrecarga objetiva, determinariam o sentimento de sobrecarga subjetiva dos familiares. O modelo inclui, ainda, o efeito de fatores moduladores sobre o grau de sobrecarga subjetiva, tais como a qualidade da relação existente entre o paciente e seus familiares e o suporte social recebido pelos familiares, podendo amenizar ou agravar a sobrecarga subjetiva sentida pelos cuidadores ${ }^{10}$. Um bom relacionamento do paciente com o familiar pode amenizar o sentimento de sobrecarga subjetiva. Entre as variáveis desse modelo, será focalizado, neste trabalho, o diagnóstico dos pacientes e sua relação com a sobrecarga dos familiares cuidadores.

Na revisão de literatura de Maurin e Boyd, foi apontada a excessiva homogeneidade dos diagnósticos dos pacientes nos estudos sobre a sobrecarga dos cuidadores, que tem sido quase sempre de esquizofrenia, o que dificulta a identificação da real influência dos diferentes tipos de diagnóstico na sobrecarga dos familiares. Essa homogeneidade de diagnóstico dos pacientes impede a comparação dos resultados da sobrecarga dos cuidadores de pacientes com diferentes diagnósticos ${ }^{10,11}$.

Foi realizada uma busca sistemática nas bases indexadas dos periódicos científicos Medline, Lilacs, SciELO, PsycINFO e ISI Web of Knowledge, utilizando-se os descritores: "diagnóstico", "sobrecarga familiar", "sobrecarga do cuidador", "carga de la familia relacionada com el diagnóstico", "family burden", "diagnostic", "overload", "mental health". Foram encontrados apenas seis estudos internacionais, que investigaram a sobrecarga de familiares cuidadores de pacientes com diferentes diagnósticos psiquiátricos. Foi observada uma contradição dos resultados dessas pesquisas no que se refere ao tipo de diagnóstico dos pacientes que estaria associado a um maior grau de sobrecarga dos familiares cuidadores, sendo, portanto, necessário maior esclarecimento sobre essa temática. Observou-se, ainda, carência 
de estudos sobre a relação entre essas variáveis. O presente trabalho teve por objetivo avaliar a sobrecarga dos familiares cuidadores em relação a dois tipos de diagnóstico de pacientes psiquiátricos: esquizofrenia e depressão maior. Os principais diagnósticos utilizados nas pesquisas sobre sobrecarga familiar foram esquizofrenia e depressão. O uso desses diagnósticos possibilitou a comparação dos resultados encontrados nos diferentes estudos com o presente trabalho.

\section{MÉTODOS}

\section{Participantes}

A população-alvo desta pesquisa foi composta pelos familiares cuidadores de pacientes atendidos no CAPS da cidade de Barbacena e no Ambulatório do Centro Hospitalar Psiquiátrico de Barbacena, em Minas Gerais, Brasil. Foram selecionadas, por meio da técnica de amostragem probabilística, duas amostras de acordo com os diagnósticos dos pacientes, de esquizofrenia e de depressão maior. Os diagnósticos foram obtidos nos prontuários dos serviços e se baseavam na Classificação Internacional de Doenças (CID-10), totalizando 123 pacientes com esquizofrenia e 407 pacientes com depressão maior.

Foi utilizada uma amostra total de 100 familiares, dos quais 50 cuidavam de pacientes com esquizofrenia e 50 cuidavam de pacientes com depressão maior. O tamanho da amostra foi determinado por cálculos estatísticos ${ }^{12}$, visando a um poder da amostra de $90 \%{ }^{13,14}$. Foram repostos 22 familiares de pacientes com esquizofrenia e 16 familiares de pacientes com depressão por não aceitarem ou por impossibilidade de participar da pesquisa. Para maior confiabilidade dos diagnósticos, foi feita uma análise adicional da descrição do quadro clínico de $20 \%$ dos pacientes da amostra por um psiquiatra independente.

\section{Instrumentos de medida}

Foram utilizados dois instrumentos de medida. O grau de sobrecarga dos familiares foi avaliado por meio da Escala de Sobrecarga dos Familiares de Pacientes Psiquiátricos (FBIS-BR), cuja escala original de Tessler e Gamache foi adaptada e validada para o Brasil ${ }^{7,15}$. A FBIS-BR avalia a sobrecarga objetiva e subjetiva dos familiares por meio de 52 itens distribuídos em cinco subescalas: (1) Assistência na vida cotidiana; (2) Supervisão aos comportamentos problemáticos; (3) Gastos; (4) Impacto nas rotinas diárias; (5) Preocupações com o paciente. A sobrecarga objetiva é avaliada pela frequência de assistências fornecidas pelos familiares ao paciente na vida cotidiana, de supervisões aos seus comportamentos problemáticos e do impacto na rotina diária do familiar cuidador, utilizando-se escalas do tipo Likert de cinco pontos ( 1 = nenhuma vez até $5=$ todos os dias). A sobrecarga subjetiva é avaliada com base em escalas do tipo Likert de quatro pontos para o grau de incômodo sentido pelos familiares nas tarefas cotidianas e supervisões dos comportamentos problemáticos ( $1=$ nem um pouco até $4=$ muito) e por escalas de cinco pontos ( $1=$ nunca até $5=$ sempre ou quase sempre) para a frequência de preocupações e peso financeiro sentido pelos familiares. A escala FBIS-BR encontra-se disponibilizada na página da internet: http://www.ufsj.edu.br/lapsam.

Os dados sociodemográficos e clínicos dos participantes foram coletados por meio de um questionário elaborado no Laboratório de Pesquisa em Saúde Mental (LAPSAM) da Universidade Federal de São João del-Rei (UFSJ), com base na literatura da área. Esse questionário foi submetido a um estudo piloto que visou avaliar sua adequação e facilidade de compreensão pela população-alvo estudada.

\section{Coleta de dados}

Após aprovação da Comissão de Ética em Pesquisa Envolvendo Seres Humanos (Cepes) da Universidade Federal de São João del-Rei (UFSJ), do Comitê de Ética em Pesquisa (CEP) da FHEMIG e da Prefeitura de Barbacena, foram selecionados os participantes e realizadas as entrevistas de coleta. Os sujeitos foram informados dos objetivos e procedimentos da pesquisa e assinaram o Termo de Consentimento Livre e Esclarecido, confirmando seu acordo em participar da pesquisa. Os instrumentos de medida foram aplicados em entrevistas individuais realizadas por estagiários de Psicologia previamente treinados, na residência dos participantes, em horários previamente combinados com os familiares, segundo suas disponibilidades.

\section{Análise dos dados}

Para caracterizar cada grupo de cuidadores, foram feitos cálculos de porcentagens, médias, desvios-padrão e valores mínimo e máximo das variáveis sociodemográficas, clínicas e de condições de vida. Foi realizada uma análise intragrupo para caracterizar o nível de sobrecarga e as dimensões da sobrecarga mais elevadas em cada grupo de cuidadores, por meio do cálculo de médias e desvios-padrão e do teste t de Student para amostras pareadas.

Foram feitas análises intergrupos para identificar diferenças significativas no grau de sobrecarga entre os dois grupos de cuidadores, para os escores globais, as subescalas e os itens específicos da FBIS-BR, utilizando-se o teste t de Student para amostras independentes. Foi feita, ainda, uma análise estatística univariada, com o teste t de Student para amostras independentes, visando identificar as variáveis sociodemográficas e de condições de vida que diferenciavam os dois grupos, para selecionar aquelas que seriam utilizadas nas análises multivariadas. A descrição dos dados referentes a essas análises univariadas encontra-se em Nolasco ${ }^{12}$. 
Para identificar os fatores preditores da sobrecarga dos familiares, foram realizadas análises multivariadas, de regressão linear múltipla, pelo método stepwise, para cada grupo de cuidadores. A variável dependente constituiu o grau de sobrecarga subjetiva dos familiares, e as variáveis independentes foram as variáveis sociodemográficas, clínicas e de condições de vida identificadas como significativas na análise univariada $(p<0,05)$ e também as variáveis não significativas que apresentaram o valor de $p \leq 0,25^{16}$. Segundo recomendações de alguns autores, essas variáveis não significativas devem ser incluídas no modelo de regressão linear, pois podem se tornar significativas nas análises multivariadas ${ }^{16}$. A distribuição normal das amostras foi confirmada por meio do teste de Kolmogorov-Smirnov (K-S =0,82/ $p=0,50 ; \mathrm{K}-\mathrm{S}=0,99 / p=0,27 ; \mathrm{K}-\mathrm{S}$ $=93,00 / p=0,35 ; \mathrm{K}-\mathrm{S}=1,29 / p=0,07)$. Não foram utilizadas as constantes na construção dos modelos de regressão, tendo em vista que a FBIS-BR não contém o valor zero para o grau de sobrecarga ${ }^{17}$. Adotou-se o nível de significância $p<0,05^{18}$. A probabilidade de erro foi de $5 \%$. Foi utilizado o software Statistical Program for Social Sciences (SPSS), versão 13,0.

\section{RESULTADOS}

\section{Descrição da amostra}

No grupo de familiares cuidadores de pacientes com esquizofrenia, a idade média era de 53,72 anos, sendo a maioria do gênero feminino (89\%). Quanto à escolaridade, quase todos os familiares (94\%) haviam frequentado a escola, e 58\% possuíam ensino fundamental completo ou incompleto. A maioria (67\%) era proprietária da casa onde morava e 54\% recebiam de um a dois salários-mínimos. Quanto ao parentesco, 55\% eram pais dos pacientes. Esses familiares cuidavam de pacientes com as seguintes características: idade média de 38,84 anos, sexo masculino (64\%), solteiros (72\%), com ensino fundamental completo ou incompleto (58\%). A maior parte não possuía doenças físicas (82\%), aceitava tomar a medicação (66\%), não tomava os medicamentos sozinhos (72\%), não estava em crise (96\%) e apresentou, no último ano, um número médio de 2,30 crises. Eles apresentavam, em média, 4,88 comportamentos problemáticos.

No grupo de familiares cuidadores de pacientes com depressão maior, a idade média foi de 48,52 anos, sendo a maioria do gênero feminino (86\%). Em relação à escolaridade, 96\% haviam frequentado a escola, e 52\% possuíam ensino fundamental completo ou incompleto. A maioria era proprietária da casa onde morava (66\%) e grande parte (54\%) recebia entre um e dois salários-mínimos. Quanto ao parentesco, $54 \%$ eram pais dos pacientes. Esses familiares cuidavam de pacientes com as seguintes características: sexo feminino (64\%), idade média de 37,24 anos, solteiros (34\%), com ensino fundamental completo ou incompleto
(54\%). A maioria não possuía doenças físicas (78\%), aceitava tomar medicação (92\%), tomava medicação sozinha (72\%), não estava em crise (92\%) e apresentou, no último ano, um número médio de 3,52 de crises. Eles apresentavam, em média, 3,76 comportamentos problemáticos.

A confiabilidade dos diagnósticos dos pacientes, avaliada por um psiquiatra independente, foi de $85 \%$. Apenas três pacientes não tiveram seus diagnósticos confirmados, sendo dois com esquizofrenia e um com depressão maior. Nos três casos, os familiares dos pacientes foram substituídos mediante seleção aleatória. A descrição completa dos resultados encontra-se em Nolasco ${ }^{12}$.

\section{Análises intergrupos}

A comparação dos dois grupos de familiares foi feita em três níveis de análise: (1) dos escores globais; (2) das subescalas; (3) dos itens específicos da escala de sobrecarga. Na primeira análise, os resultados mostraram que não houve diferença estaticamente significativa entre as médias dos escores globais de sobrecarga objetiva e subjetiva dos familiares, do grupo de esquizofrenia (médias/desvios-padrão $=2,09 / 0,39 ; 2,77 / 0,49$ ) e de depressão maior (médias/desvios-padrão = 1,96/0,66; $2,98 / 0,49)-p>0,05$. Na análise das subescalas, observou-se uma diferença significativa entre os dois grupos de cuidadores $(p<0,001)$ apenas para a dimensão "Assistência na vida cotidiana". Os cuidadores de pacientes com esquizofrenia apresentaram sobrecarga objetiva significativamente mais elevada nessa subescala (média/desvio-padrão $=2,68 / 0,64$ ) do que os cuidadores de pacientes com depressão maior (média/desvio-padrão =2,26/0,81), indicando que eles davam assistência mais frequente aos pacientes na vida cotidiana.

$\mathrm{Na}$ análise dos itens específicos da escala, foram observadas diferenças significativas entre os dois grupos de cuidadores (Tabela 1). No que se refere à subescala "Assistência na vida cotidiana", o grau de sobrecarga objetiva foi mais elevado para os cuidadores de pacientes com esquizofrenia, nos itens referentes à frequência da assistência prestada na tomada de medicamentos e na administração do dinheiro. O grau de sobrecarga subjetiva (sentimento de incômodo) foi maior para os cuidadores de pacientes com depressão maior, nos itens referentes à ajuda ao paciente na higiene corporal, nas compras de alimentos ou outros objetos, na administração do dinheiro e no acompanhamento em consultas médicas.

Os resultados da subescala de "Supervisão dos comportamentos problemáticos" mostraram que os cuidadores de pacientes com depressão maior apresentaram frequência significativamente mais elevada de supervisão dos comportamentos de autoagressividade do que os cuidadores de pacientes de esquizofrenia. No que se refere à subescala "Preocupações com o paciente", os familiares cuidadores de pacientes com depressão maior apresentaram frequência mais elevada de preocupações com a vida social dos pacientes do que o outro grupo de cuidadores. 
Tabela 1. Médias e desvios-padrão dos escores de sobrecargas objetiva (a) e subjetiva (b) para os itens que apresentaram diferenças significativas entre os grupos e valores de $p$

\begin{tabular}{|c|c|c|c|c|}
\hline \multicolumn{2}{|l|}{ Itens } & \multirow{2}{*}{$\begin{array}{c}\text { Diagnósticos do paciente } \\
\text { Esquizofrenia }\end{array}$} & \multirow{2}{*}{$\begin{array}{c}\text { Médias/desvios-padrão } \\
1,93(0,94)\end{array}$} & \multirow{2}{*}{$\frac{p}{0,01^{*}}$} \\
\hline Higiene & $A 1 b$ & & & \\
\hline & & Depressão maior & $2,67(1,15)$ & \\
\hline \multirow[t]{2}{*}{ Medicação } & $A 2 a$ & Esquizofrenia & $3,76(1,69)$ & $0,00^{*}$ \\
\hline & & Depressão maior & $2,40(1,86)$ & \\
\hline \multirow[t]{2}{*}{ Compras } & $A 4 b$ & Esquizofrenia & $1,52(0,72)$ & $0,00^{*}$ \\
\hline & & Depressão maior & $2,16(0,89)$ & \\
\hline \multirow[t]{2}{*}{ Transporte } & A6b & Esquizofrenia & $1,69(0,79)$ & $0,03^{*}$ \\
\hline & & Depressão maior & $1,28(0,61)$ & \\
\hline \multirow[t]{4}{*}{ Administração do dinheiro } & A7a & Esquizofrenia & $2,84(1,80)$ & $0,00^{*}$ \\
\hline & & Depressão maior & $1,50(1,23)$ & \\
\hline & $A 7 b$ & Esquizofrenia & $1,47(0,57)$ & $0,04^{*}$ \\
\hline & & Depressão maior & $2,44(1,23)$ & \\
\hline \multirow[t]{2}{*}{ Acompanhamento na consulta médica } & $A 9 b$ & Esquizofrenia & $1,50(0,85)$ & $0,003^{*}$ \\
\hline & & Depressão maior & $2,17(1,04)$ & \\
\hline \multirow[t]{2}{*}{ Comportamento autoagressivo } & B5a & Esquizofrenia & $1,22(0,58)$ & $0,00^{*}$ \\
\hline & & Depressão maior & $1,92(1,17)$ & \\
\hline \multirow[t]{2}{*}{ Vida social } & E3 & Esquizofrenia & $3,68(1,47)$ & $0,03^{*}$ \\
\hline & & Depressão maior & $4,20(0,85)$ & \\
\hline
\end{tabular}

Teste t de Student; ${ }^{*} p<0,05$

\section{Análises intragrupo}

Os resultados da análise intragrupo indicaram quais dimensões da sobrecarga eram mais elevadas em cada grupo de cuidadores. Observou-se que ambos os grupos apresentavam maior grau de sobrecarga objetiva na subescala "Assistência na vida cotidiana", indicando que havia frequência maior de tarefas de assistência ao paciente na vida cotidiana do que das demais atividades de cuidar. Por outro lado, foi observado maior grau de sobrecarga subjetiva para a subescala "Supervisão dos comportamentos problemáticos", indicando que ambos os grupos sentiam maior grau de incômodo ao supervisionarem os comportamentos problemáticos dos pacientes do que ao desempenharem as demais atividades de cuidar.

\section{Análises multivariadas: fatores preditores da sobrecarga subjetiva}

Foram realizadas, para cada grupo de cuidadores, quatro análises de regressão da sobrecarga subjetiva para as seguintes categorias de variáveis: (1) características sociodemográficas dos familiares; (2) condições de vida dos familiares; (3) características sociodemográficas dos pacientes; (4) características clínicas dos pacientes.

\section{Fatores preditores no grupo com esquizofrenia}

A tabela 2 apresenta os resultados desse grupo de cuidadores. No que se refere às características sociodemográficas dos familiares, os fatores preditores da sobrecarga subjetiva foram: pessoas mais velhas, que tinham menor grau de escolaridade (ensino fundamental ou analfabeto), que não eram pais do paciente (eram irmãos, cônjuges, filhos ou outro tipo de parentesco) e não possuíam renda. Quanto às condições de vida dos familiares, os fatores preditores de maior sobrecarga subjetiva foram: estar fazendo tratamento de saúde, ter maior número de filhos e ter outras preocupações na vida.

A análise das características sociodemográficas dos pacientes mostrou que o grau de sobrecarga subjetiva dos familiares era maior quando o paciente era mais velho, do sexo masculino e não possuía renda. No que se refere às características clínicas e de comportamento dos pacientes, os fatores preditores de maior sobrecarga subjetiva dos familiares foram: o paciente estar em crise, ter apresentado maior número de crises no último ano, apresentar maior número de comportamentos problemáticos e ter menor duração do tratamento.

\section{Fatores preditores no grupo com depressão maior}

A tabela 3 apresenta os resultados desse grupo de cuidadores. No que se refere às características sociodemográficas dos familiares, a sobrecarga subjetiva era maior quando o familiar era do sexo feminino, tinha maior nível de escolaridade (ensino fundamental completo, ensino médio completo ou incompleto, graduação e pós-graduação) e não possuía 
renda. Em relação às condições de vida dos familiares, os fatores preditores de maior grau de sobrecarga foram: o familiar ter mais pessoas morando com ele, possuir mais pessoas doentes sob seu cuidado, ter outras preocupações na vida, não praticar atividades de lazer e não trabalhar.

Quanto às características sociodemográficas dos pacientes, observou-se que a sobrecarga subjetiva dos familiares era maior quando o paciente não possuía atividade fora de casa, tinha maior nível de escolaridade, não tinha um quarto individual e não era proprietário da residência. A análise das características clínicas dos pacientes mostrou os seguintes fatores preditores: apresentar comorbidades, tomar maior quantidade de medicamentos, apresentar doenças físicas e apresentar o transtorno psiquiátrico há mais tempo.

Tabela 2. Análises de regressão múltipla das características dos familiares e pacientes associadas à sobrecarga global subjetiva, no grupo de familiares cuidadores de pacientes com esquizofrenia

\begin{tabular}{|c|c|c|c|c|c|c|}
\hline Categorias & Variáveis & Beta & Erro-padrão & $t$ & $p$ & \\
\hline \multirow[t]{4}{*}{ Características sociodemográficas dos familiares } & Idade & 0,95 & 0,00 & 10,39 & 0,00 & \multirow{4}{*}{$\begin{array}{c}\mathrm{R}^{2}=0,92 \\
\mathrm{~F}(4,46)=136,44 \\
(\mathrm{p}=0,00) \\
{[\mathrm{DW}=1,83]}\end{array}$} \\
\hline & Escolaridade & $-0,13$ & 0,28 & $-1,64$ & 0,11 & \\
\hline & Possui renda & 0,10 & 0,29 & 2,14 & 0,03 & \\
\hline & Parentesco entre familiar e paciente & 0,12 & 0,21 & 2,47 & 0,01 & \\
\hline \multirow[t]{3}{*}{ Condições de vida dos familiares } & Número de filhos & 0,42 & 0,07 & 5,49 & 0,00 & \multirow{3}{*}{$\begin{array}{c}\mathrm{R}^{2}=0,79 \\
\mathrm{~F}(3,47)=62,73 \\
(\mathrm{p}=0,00) \\
{[\mathrm{DW}=1,69]}\end{array}$} \\
\hline & Outras preocupações & 0,23 & 0,35 & 3,19 & 0,00 & \\
\hline & Tratamento para saúde & 0,48 & 0,30 & 6,49 & 0,00 & \\
\hline \multirow[t]{3}{*}{ Características sociodemográficas dos pacientes } & Idade & 0,68 & 0,00 & 12,64 & 0,00 & \multirow{3}{*}{$\begin{array}{c}\mathrm{R}^{2}=0,93 \\
\mathrm{~F}(3,47)=219,36 \\
(\mathrm{p}=0,00) \\
{[\mathrm{DW}=2,15]}\end{array}$} \\
\hline & Sexo & 0,27 & 0,19 & 4,97 & 0,00 & \\
\hline & Possui renda & 0,15 & 0,23 & 3,50 & 0,00 & \\
\hline \multirow[t]{4}{*}{ Características clínicas dos pacientes } & Número de crises no último ano & 0,19 & 0,03 & 4,82 & 0,00 & \multirow{4}{*}{$\begin{array}{c}\mathrm{R}^{2}=0,95 \\
F(4,46)=241,61 \\
(p=0,00) \\
{[D W=1,89]}\end{array}$} \\
\hline & Duração do tratamento (em anos) & $-0,08$ & 0,01 & $-1,50$ & 0,14 & \\
\hline & Número de comportamentos problemáticos & 0,12 & 0,03 & 1,97 & 0,05 & \\
\hline & Presença de crise atual & 0,83 & 0,21 & 11,39 & 0,00 & \\
\hline
\end{tabular}

Tabela 3. Análises de regressão múltipla das características dos familiares e pacientes associadas à sobrecarga global subjetiva no grupo de familiares cuidadores de pacientes com depressão maior

\begin{tabular}{|c|c|c|c|c|c|c|}
\hline Categorias & Variáveis & Beta & Erro-padrão & $t$ & $p$ & \\
\hline \multirow[t]{3}{*}{ Características sociodemográficas dos familiares } & Sexo & 0,70 & 0,24 & 9,49 & 0,00 & \multirow{3}{*}{$\begin{array}{c}\mathrm{R}^{2}=0,86 \\
\mathrm{~F}(3,47)=102,69 \\
(\mathrm{p}=0,00) \\
{[\mathrm{DW}=1,74]}\end{array}$} \\
\hline & Possui renda & 0,16 & 0,40 & 2,76 & 0,01 & \\
\hline & Escolaridade & 0,23 & 0,29 & 3,44 & 0,00 & \\
\hline \multirow[t]{5}{*}{ Condições de vida dos familiares } & $\begin{array}{l}\text { Número de pessoas que moram com } 0 \\
\text { familiar }\end{array}$ & 0,68 & 0,06 & 9,61 & 0,00 & \multirow{5}{*}{$\begin{array}{c}R^{2}=0,92 \\
F(5,45)=114,18 \\
(p=0,00) \\
{[D W=1,47]}\end{array}$} \\
\hline & $\begin{array}{l}\text { Número de outros familiares que precisam } \\
\text { de cuidados }\end{array}$ & 0,20 & 0,17 & 4,67 & 0,00 & \\
\hline & Trabalho & 0,09 & 0,25 & 1,58 & 0,12 & \\
\hline & Outras preocupações & 0,11 & 0,31 & 2,45 & 0,02 & \\
\hline & Lazer fora de casa & 0,11 & 0,26 & 1,58 & 0,10 & \\
\hline \multirow[t]{4}{*}{ Características sociodemográficas dos pacientes } & Escolaridade & 0,27 & 0,51 & 2,61 & 0,01 & \multirow{4}{*}{$\begin{array}{c}R^{2}=0,65 \\
F(4,46)=24,48 \\
(p=0,00) \\
{[D W=1,11]}\end{array}$} \\
\hline & Atividade fora de casa & 0,30 & 0,54 & 2,53 & 0,01 & \\
\hline & Tipo de residência & 0,15 & 0,63 & 1,47 & 0,14 & \\
\hline & Quarto individual & 0,28 & 0,54 & 2,28 & 0,03 & \\
\hline \multirow[t]{4}{*}{ Características clínicas dos pacientes } & Duração da doença (em anos) & 0,17 & 0,02 & 2,94 & 0,00 & \multirow{4}{*}{$\begin{array}{c}R^{2}=0,93 \\
F(4,46)=164,61 \\
(p=0,00) \\
{[D W=1,35]}\end{array}$} \\
\hline & Quantidade de medicamentos & 0,41 & 0,07 & 5,91 & 0,00 & \\
\hline & Presença de doenças físicas & 0,22 & 0,29 & 4,32 & 0,00 & \\
\hline & Presença de comorbidades & 0,42 & 0,20 & 8,48 & 0,00 & \\
\hline
\end{tabular}




\section{DISCUSSÃO}

Os resultados obtidos permitiram identificar semelhanças e diferenças entre os dois grupos de familiares avaliados. No que se refere às semelhanças, para ambos os grupos, a sobrecarga objetiva foi maior para a frequência das tarefas de assistência cotidiana do que as demais atividades de cuidar. Quanto à sobrecarga subjetiva, para ambos os grupos, supervisionar os comportamentos problemáticos dos pacientes gerava maior grau de incômodo do que as demais atividades. Na avaliação do impacto causado na rotina diária do familiar e nos escores globais de sobrecarga, não houve diferença significativa entre os dois grupos. Esses resultados confirmam dados de outras pesquisas ${ }^{19-23}$.

As diferenças encontradas entre os grupos para o grau de sobrecarga se referem às análises realizadas por subescalas e por itens específicos do instrumento utilizado. No que se refere à assistência cotidiana, os cuidadores de pacientes com esquizofrenia apresentaram maior sobrecarga objetiva com relação à tomada dos medicamentos. Eles ajudaram ou lembraram os pacientes mais frequentemente a tomarem os remédios, tendo algumas vezes que os administrar pessoalmente ou às escondidas. Os familiares de pacientes com depressão maior relataram sentir mais incômodo ao prestar assistência aos pacientes para a higiene corporal, as compras de alimentos ou outros objetos, a administração do dinheiro e o acompanhamento em consultas médicas. Esses familiares também apresentaram frequência mais elevada de supervisão dos comportamentos autoagressivos. Os estudos identificados na literatura não possuíam esse tipo de dados, não permitindo a comparação entre eles.

O sentimento de peso financeiro que o paciente ocasionou foi significativamente mais elevado em familiares cuidadores de pacientes com esquizofrenia, corroborando os achados de outros estudos ${ }^{23,24}$. No que se refere às preocupações com os pacientes, os cuidadores de pacientes com depressão maior preocuparam-se mais frequentemente com a vida social dos pacientes, provavelmente porque a maioria permanecia em casa o dia todo, tendo pouco contato com outras pessoas. Não foram encontrados estudos para comparação desses dados.

Os fatores preditores da sobrecarga diferiram entre os dois grupos de cuidadores. No grupo de cuidadores de pacientes com esquizofrenia, as variáveis dos familiares referentes à baixa escolaridade e a maior idade foram preditoras de maior grau de sobrecarga, corroborando os achados citados em uma revisão de estudos? ${ }^{7}$ Com relação ao grau de parentesco, os cuidadores que eram irmãos, cônjuges e filhos dos pacientes apresentaram maior sentimento de incômodo com a tarefa de cuidar. Segundo Tessler e Gamache, a distribuição dos cuidados pelos membros das famílias espelha o típico padrão de obrigação segundo o parentesco, sendo os pais e os cônjuges os mais envolvidos nessa tarefa em re- lação aos demais familiares. Os autores destacaram que os irmãos não gostam de cuidar, presumivelmente porque isso interfere na sua própria trajetória de vida, já que eles passam a ter obrigações que normalmente não estão associadas com o papel de um irmão, e, sim, com o dos pais ${ }^{5,7}$. Porém, esses dados contrastaram com os resultados de uma pesquisa realizada na Espanha, na qual a sobrecarga foi maior para pais e cônjuges ${ }^{25}$. Essa contradição talvez se explique por diferenças culturais entre as amostras estudadas nesses países. Dois outros fatores preditores da sobrecarga que confirmaram dados de outras pesquisas foram: a baixa renda dos familiares ${ }^{26}$ e ter maior número de filhos ${ }^{27}$. Os familiares estarem fazendo tratamento de saúde e terem outras preocupações na vida foram também dois preditores da sobrecarga, confirmando dados de outras pesquisas 9,17,28. $^{2}$.

No que se refere às variáveis dos pacientes, ainda no grupo de esquizofrenia, o sexo masculino e não possuir renda foram dois fatores preditores de sobrecarga dos familiares, corroborando outros estudos $25,27,29$. O número de comportamentos problemáticos dos pacientes também foi um fator preditor da sobrecarga, em conformidade com dados de outras pesquisas ${ }^{24,27,30}$ e de estudos citados em três revisões de literatura ${ }^{7,10,11}$. Esses autores também encontraram que a presença de crise atual, o número de crises e a duração do tratamento estavam significativamente relacionados à sobrecarga do familiar, confirmando dados do presente estudo, no qual esses fatores foram preditores da sobrecarga.

No grupo de cuidadores de pacientes com depressão, as variáveis sociodemográficas dos familiares associadas à sobrecarga subjetiva foram: o sexo feminino e o maior nível de escolaridade. Os familiares do sexo feminino acumulam excesso de tarefas, com os afazeres domésticos e os cuidados aos pacientes, o que aumenta sua sobrecarga ${ }^{5}$. Fazer essas atividades tende a ser pensado como um trabalho das mulheres. Quanto aos afazeres domésticos e ao preparo da alimentação, a maneira como os membros da família percebem a necessidade de ajudar em relação aos afazeres domésticos pode estar relacionada com os papéis divididos por gênero, e os familiares do sexo feminino percebem maior necessidade de fornecer ajuda nesse domínio do que os familiares do sexo masculino ${ }^{5}$. O resultado referente ao nível de escolaridade confirmou dados de outro estudo ${ }^{31}$. No que se refere às condições de vida dos familiares, os fatores preditores encontrados foram: ter pessoas doentes em casa, ter outras preocupações na vida, não praticar atividades de lazer e não trabalhar. Esses resultados estão em acordo com dados de outros estudos 22,25,28,30.

Quanto às variáveis das características dos pacientes com depressão, um dos fatores preditores da sobrecarga foi o fato de o paciente não possuir um quarto individual, que resulta em falta de privacidade e incômodo para os familiares ${ }^{22}$. Outros fatores preditores da sobrecarga, que confirmaram dados 
de outras pesquisas foram: o paciente não exercer uma atividade fora de $\operatorname{casa}^{25}$, ter doenças físicas ${ }^{19,32}$, tomar muitos medicamentos ${ }^{19,22,33}$ e ter maior duração do transtorno psiquiátrico $7,23,25,28$. Esses dados sugerem que a gravidade do quadro clínico dos pacientes está associada ao grau de sobrecarga, confirmando resultados de diversas pesquisas $7,10,11,29,33,34$.

\section{CONCLUSÃO}

Os resultados deste estudo apontaram para diferenças que caracterizam como os familiares dos dois grupos reagem ao papel de cuidador e as dificuldades diferenciadas que apresentam no enfrentamento dessa situação, no caso dos cuidadores de pacientes com esquizofrenia e com depressão maior. O reconhecimento dessas diferenças permite identificar as necessidades específicas dos cuidadores em relação ao diagnóstico do paciente, podendo, assim, direcionar o trabaIho dos profissionais dos serviços de saúde mental. Estudos futuros devem investigar as características da sobrecarga de familiares cuidadores de pacientes com outros diagnósticos psiquiátricos, além dos dois tipos avaliados neste trabalho, contribuindo, assim, para o aumento do conhecimento diferenciado sobre a sobrecarga dos familiares. Além do uso Escala de Sobrecarga dos Familiares de Pacientes Psiquiátricos, trabalhos futuros poderiam incluir escalas de avaliação de sintomas de depressão e ansiedade nos familiares, buscando relações entre esses constructos e a sobrecarga familiar.

\section{CONTRIBUIÇÕES INDIVIDUAIS}

Marcela Nolasco - Contribuiu substancialmente na execução e no planejamento do projeto, na obtenção dos dados e na análise e interpretação dos dados.

Marina Bandeira - Contribuiu na concepção e no planejamento do projeto, na interpretação dos dados e na revisão crítica de conteúdo.

Marcos Santos de Oliveira - Contribuiu na análise e interpretação dos dados.

Carlos Eduardo Leal Vidal - Participou da obtenção dos dados.

Todos os autores deste artigo participaram diretamente no planejamento, execução ou análise dos dados deste estudo.

Todos os autores deste artigo leram e aprovaram a versão final enviada.

O conteúdo deste manuscrito não teve os direitos autorais concedidos e não foi publicado anteriormente.

O conteúdo deste manuscrito não se encontra sob consideração para publicação em nenhuma outra revista no momento.

O conteúdo deste manuscrito não terá os direitos autorais concedidos, bem como não será submetido ou publica- do em nenhuma outra revista enquanto a aceitação pelos editores do periódico estiver sob consideração.

Não há manuscritos ou resumos diretamente relacionados com o assunto deste trabalho publicados ou não publicados por qualquer autor deste artigo.

\section{CONFLITOS DE INTERESSE}

Marcela Nolasco, Marina Bandeira, Marcos Santos de Oliveira e Carlos Eduardo Leal Vidal não possuem conflitos de interesse a serem declarados.

\section{AGRADECIMENTOS}

Aos amigos e colegas do LAPSAM e da UFSJ, aos Serviços de Saúde Mental de Barbacena (FHEMIG e CAPS), por acolherem esta pesquisa, tornando possível a sua realização e aos familiares dos pacientes psiquiátricos que participaram das entrevistas.

\section{REFERÊNCIAS}

1. Morgado A, Lima LB. Desinstitucionalização: suas bases e a experiência internacional. J Bras Psiquiatr. 1994;43(1):19-28.

2. Banderia M. Desinstitucionalização ou transistitucionalização: lições de alguns países. J Bras Psiquiatr. 1991;40(7):355-60.

3. Bandeira M, Gelinas D, Lesage A. Desinstitucionalização: o programa de acompanhamento intensivo na comunidade. J Bras Psiquiatr. 1998;47(12):627-40.

4. Hanson JG, Rapp CA. Families' perceptions of community mental health programs for their relatives with a severe mental illness. Community Ment Health. 1992;28(3):181-97.

5. Tessler RC, Gamache GM. Family experiences with mental illness. Westport: Auburn House; 2000.

6. Hatfield AB. What families want of family therapists. In: MacFarlane WR (Ed.). Family therapy in schizophrenia. New York: Guilford Press; 1983. p. 41-65.

7. Bandeira M, Barroso S. Sobrecarga das famílias de pacientes psiquiátricos. J Bras Psiquiatr. 2005;54(1):34-46.

8. Soares $(B$, Munari DB. Considerações acerca da sobrecarga em familiares de pessoas com transtornos mentais. Ciênc Cuid Saúde. 2007;6(3):357-62.

9. Borba LO, SchwartzE, Kantorski LP. A sobrecarga da família que convive com a realidade do transtorno mental. Acta Paul Enferm. 2008;21(4):588-94.

10. Maurin TJ, Boyd CB. Burden of mental illness on the family: a critical review. Arch Psychiatr Nurs. 1990;2:99-107.

11. Rose LE. Families of psychiatric patients: a critical review and future research directions. Arch Psychiatr Nurs. 1996;10:67-76.

12. Nolasco M. Sobrecarga dos familiares cuidadores em função do diagnóstico dos pacientes psiquiátricos [dissertação de mestrado]. São João del-Rei: Universidade Federal de São João del-Rei; 2013.

13. Snedecor GW, Cochran WG. Métodos estadísticos. México: Cia. Ed. Continental; 1971.

14. Resende MDV, Sturion JA, Pereira JCD. Tamanho amostral para detecção de diferenças significativas entre tratamentos. Bol Pesq Fl. 2004;49:109-20.

15. Bandeira M, Calzavara MGP, Castro I. Estudo de validade da escala de sobrecarga de familiares cuidadores de pacientes psiquiátricos. J Bras Psiquiatr. 2008;57(2):98-104.

16. Hosmer DW, Lemeshou S. Applied logistic regression. New York: John Wiley and Sons; 2000. 
17. Montgomery DC, Peck EA. Introduction to linear regression analysis. 2nd ed. New York: John Wiley \& Sons; 1992. p. 290-8.

18. Levin J. Estatística aplicada a ciências humanas. 2a ed. São Paulo: Harbra; 1987.

19. Batista CMF. Sobrecarga de familiares de pacientes psiquiátricos: influência do gênero do cuidador [dissertação de mestrado]. São João del-Rei: Universidade Federal de São João del-Rei; 2012.

20. Albuquerque EPT, Cintra AMO, Bandeira M. Sobrecarga de familiares de pacientes psiquiátricos: comparação entre diferentes tipos de cuidadores. J Bras Psiquiatr. 2010;59(4):308-16.

21. Albuquerque EPT. Sobrecarga de familiares de pacientes psiquiátricos: estudo de diferentes tipos de cuidadores [dissertação de mestrado]. São João del-Rei: Universidade Federal de São João del-Rei; 2010.

22. Barroso S, Bandeira M, Nascimento E. Sobrecarga de familiares de pacientes psiquiátricos atendidos na rede pública. Rev Psiq Clín. 2007;34(6):270-7.

23. Hadrys T, Adamowski T, Kiejna A. Mental disorder in Polish families: is diagnosis a predictor of caregiver's burden? Soc Psychiatry Psychiatr Epidemiol. 2011;46:363-72.

24. Wijngaarden BV, Schene AH, Koeter MWJ. Family caregiving in depression: impact on caregivers' daily life, distress, and help seeking. J Affect Disord. 2004;81:211-22.

25. Martínez A, Nadal S, Beperet ME, Mendióroz P. Sobrecarga de los cuidadores familiares de pacientes con esquizofrenia: factores determinantes. Anales Sis San Navarra. 2000;23(1):101-10
26. Severo AKS, Dimenstein M, Brito M, Cabral C, Alverga AR. A experiência de familiares no cuidado em saúde mental. Arq Bras Psicol. 2007;59:143-55.

27. Barroso S, Bandeira M, Nascimento E. Fatores preditores de sobrecarga subjetiva de familiares de pacientes psiquiátricos atendidos na rede pública de Belo Horizonte, Minas Gerais, Brasil. Cad Saude Publica. 2009;25(9):1957-68.

28. Hasui C, Sakomoto S, Sugiura T, Miyata R, Fugil Y, Koshiisshi F, et al. Burden on family members of the mentally ill: a naturalistic study in Japan. Compr Psychiatry. 2002;43(3):219-22.

29. Awad AG, Voruganti LNP. The burden of schizophrenia on caregivers: a review. Pharmacoeconomics. 2008;26(2):149-62.

30. Möller-Leimkühler AM. Burden of relatives and predictors of burden. Baseline results from the Munich 5-year-follow-up study on relatives of first hospitalized patients with schizophrenia or depression. Eur Arch Psychiatry Clin Neurosci. 2005;255:223-31.

31. Bulger MW, Wandersman A, Goldman CR. Burdens and gratifications of caregiving: appraisal parental care of adults with schizophrenia. Am J Orthopsychiatry. 1993;63:255-65.

32. Garrido R, Menezes PR. Impacto em cuidadores de idosos com demência atendidos em um serviço psicogeriátrico. Rev Saúde Pública, 2004;38(6):835-41.

33. Jenkins JH, Schumacher JG. Family burden of schizophrenia and depressive illness. Specifying the effects of ethnicity, gender and social ecology. Br J Psychiatry. 1999;174:31-8.

34. Loukissa D. Family burden in chronic mental illness: a review of research studies. J Adv Nurs. 1995;21:248-55. 